Submitted to Advanced Powder Technology

Manuscript type: Original research paper

\title{
Studies of optical properties of UV-cured acrylate films modified with spherical silica nanoparticles
}

\author{
W. Suthabanditpong ${ }^{\text {a }}$, C. Takai ${ }^{\text {a }}$, M. Fuji ${ }^{\text {a,* }}$, R. Buntem ${ }^{\text {b }}$, T. Shirai ${ }^{\text {a }}$
}
${ }^{a}$ Advanced Ceramics Research Center, Nagoya Institute of Technology, Nagoya, JAPAN
${ }^{b}$ Department of Chemistry, Faculty of Science, Silpakorn University, Nakorn Pathom, THAILAND

* To whom correspondence should be addressed: Tel: +81-572-24-8110

Fax: +81-572-24-8109

E-mail: fuji@nitech.ac.jp (M. Fuji) 


\begin{abstract}
This study presents the optical properties of UV-cured acrylate films modified with spherical silica nanoparticles. The nanoparticles together with the UV-acrylate monomer solution were first prepared by an ultrasonic method to produce a suspension of silicaacrylate. The UV-cured acrylate films modified with the nanoparticles were fabricated by coating the suspension on cleaned glass substrates, then exposed to UV light for $10 \mathrm{~min}$ at ambient temperature. The effect of the silica nanoparticle content on the optical properties of the modified films was determined by a UV-Vis spectrophotometer and an apparatus equipped with a laser. Based on the visible absorption data, the transmittance of all the modified film coated substrates is lower than that of the unmodified film. The transmittance of the modified films decreases with the increasing silica content. The increasing silica content also improves the haze and light diffusing ability of the modified films. The highest haze of about 0.26 and the largest range of scattered light were achieved using the film modified with 30 vol\% particles. These nanoparticles show a good potential to improve the optical properties of the UV-cured acrylate film, especially the light diffusing property that may be utilized in electronic display technologies.
\end{abstract}

Keywords: Silica nanoparticles, UV-cured acrylate film, Optical materials, Light diffusing property 


\section{Introduction}

There are two types of UV-curing resins, which are categorized by the curing mechanism, such as an acrylate radical polymerization and an epoxy cationic polymerization. Among these types, the UV-curable acrylate resin has a high UV-sensitivity and provides good mechanical and elastic properties such as modulus, strain hardening, tear strength, creep, and glass-rubber transition temperature after the UV-radiation curing [1-2]. For the UV-radiation curing, light hits the acrylate radical then stimulates a bonding process between molecules leading to the polymerizations. Consequently, the high cross-linked acrylate material is obtained by rapidly transforming a liquid resin into a solid material [3-4] called a UV-cured acrylate. The UV-cured acrylate is extensively used in many applications, for example, printed materials, optical surface protections, paints, durable ink, and acrylic sheets used for electronic display technologies [5-8]. In the case of the acrylic sheet, it exhibits a good transparency in the visible light range but it has a very low light diffusivity. The property of the light diffusing film is its homogeneous brightness by spreading the light that is essential for electronic display technologies to form excellent final images [9]. Many efforts have been devoted to increasing the light transmittance of the UV-cured polymer films along with increasing their light diffuse transmittance [8-10]. A variety of inorganic particles including titanium dioxide, copper oxide, aluminum hydroxide, alumina and silica are extensively used for the fabrication of light diffusing films due to their unique and enhanced mechanical, thermal, electrical and optical properties [8-11]. Among these materials, silica nanoparticles are very attractive to improve the optical properties of the polymer film because of their good light transmittance and diffusion capacity, which may be promising candidates for light diffusing films in the electronic display industry [12-13]. For these reasons, we have recently developed UV-cured acrylate films modified by spherical silica nanoparticles 
(SSNPs) with a high transparency and light diffusing property. The films were coated on a glass substrate using a bar coating machine. The transmittance and haze of all the modified films were analyzed by a UV-visible spectrophotometer and compared to the unmodified film coated on a glass substrate in the visible range $(400-800 \mathrm{~nm})$. The film having a higher haze value normally exhibits a better light diffusing ability [12]. Therefore, the light diffusing ability of the films was examined by an apparatus equipped with a $532 \mathrm{~nm}$ laser. The influence of silica content on the optical properties of the films was also investigated.

\section{Experimental}

\subsection{Materials}

The spherical silica nanoparticles (SSNPs) were purchased from Admatechs, Japan. The UV-curable acrylate monomer was supplied by the JSR Corporation, Japan. Methyl isobutyl ketone (MIBK; 99.5\%), ethanol and acetone were purchased from Wako Pure Chemical Industries, Japan. All the glass substrates used in this study were ultrasonically cleaned in deionized water, ethanol and acetone for $10 \mathrm{~min}$ in each solvent to remove any contaminants. All solvents and chemicals were of reagent grade and used as received.

\subsection{Fabrication of UV-cured acrylate films modified with spherical silica nanoparticles}

The UV-curable acrylate monomer used in this study was composed of two major components such as dipentaerythritol penta-/hexa acrylate that acted as an acrylic monomer and Irgacure 184 (1-hydroxycyclohexyl phenyl ketone) that acted as a photo initiator. The monomer was diluted in the MIBK to $20 \mathrm{vol} \%$, then mixed with a certain amount of the SSNPs by the ultrasonic method. The suspension was coated on the cleaned glass substrates using a K101 control coater (RK PrintCoat Instruments, Ltd., United Kingdom). The coater 
using a standard meter bar having a $0.51 \mathrm{~mm}$ wire diameter was set at the constant speed of $2.0 \mathrm{~cm} / \mathrm{s}$. The obtained wet silica-acrylate film was stored in the dark at room temperature for $24 \mathrm{~h}$ to evaporate the solvent contained in the film. The dry silica-acrylate film was exposed to a $110 \mathrm{~W}$ UV lamp (254 nm) in a UV-photo surface processor (PL16-110D; SEN LIGHTS Corp., Japan) at ambient temperature. A variety of silica contents in the UV-cured acrylate films was fabricated by mixing different amounts of the SSNPs in the 20 vol\% UVcurable acrylate monomer. The silica contents of the UV-cured acrylate films were 1, 5, 10, 20 and 30 vol\%.

\subsection{Characterization}

Thicknesses of all the dry and cured films were measured by a confocal laser scanning microscope (CLSM; LEXT 3D Measuring Laser Microscope CLS-4000; Olympus, Japan). Dispersibility of the SSNPs on the surface of the UV-cured acrylate films with the various silica contents was also observed by the CLSM. A field emission scanning electron microscope (FESEM; JSM7600F; JEOL, Japan) was employed to observe the morphologies of the SSNPs. The FESEM was also used to observe the morphologies of the films. Prior to imaging by the FESEM, the films were directly dried on a hot plate at a temperature of $150^{\circ} \mathrm{C}$ for $2 \mathrm{~h}$ in order to eradicate any unclear images caused by some residual solvent. The dry samples were then coated by a thin layer of osmium tetroxide using an osmium plasma coater (OPC60A; Filagen). The coated samples were subjected to FESEM in order to observe their morphologies in the COMPO mode. The transmittance and haze of the films were measured by a UV-Vis-NIR spectrophotometer (UV3150; SHIMADZU, Japan). The haze value is defined as the ratio between the diffuse transmittance and total transmittance (Haze $=$ $\mathrm{T}_{\text {diffuse }} / \mathrm{T}_{\text {total }}$ [14]. The haze value of the films was calculated at $532 \mathrm{~nm}$ corresponding to the wavelength of the laser used in the light diffusing study. The film having a higher haze value 
exhibits a better light diffusing ability [12]. The light diffusing ability of the films was examined by an apparatus equipped with a light source. The light source is a laser lamp with an emission of $532 \mathrm{~nm}$ (Z1M18B-F-532-PZ, Z-Laser, Germany). The films were placed in front of the laser light and the scattered light by the particles in the different films was obtained by a digital camera. The apparatus is illustrated by the schematic diagram in Fig. 1 .

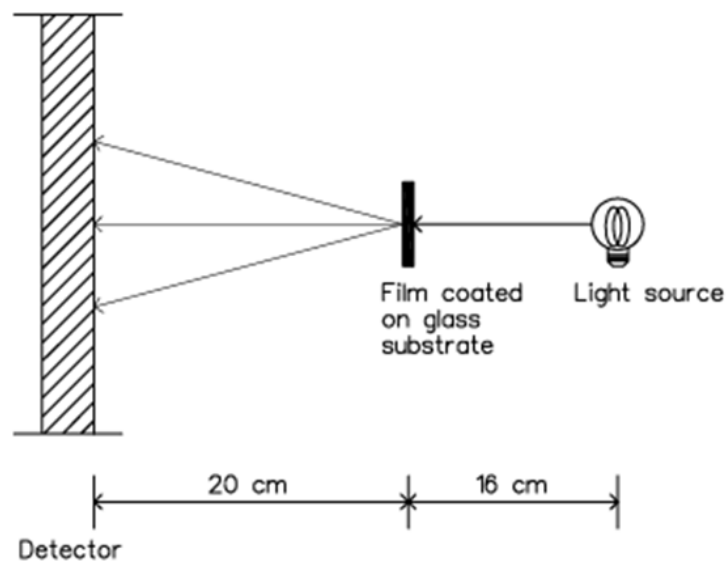

Fig. 1. Schematic illustration of the apparatus for achieving a scattered light image.

\section{Results and Discussion}

\subsection{Morphologies of UV-cured acrylate films modified with spherical silica nanoparticles}

The UV-cured acrylate films modified with the SSNPs were successfully fabricated after subjecting the dry films to UV illumination for 10 min to induce the crosslinking polymerization. The film thicknesses of the cured films were then measured by the CLSM and compared to the dry films. 


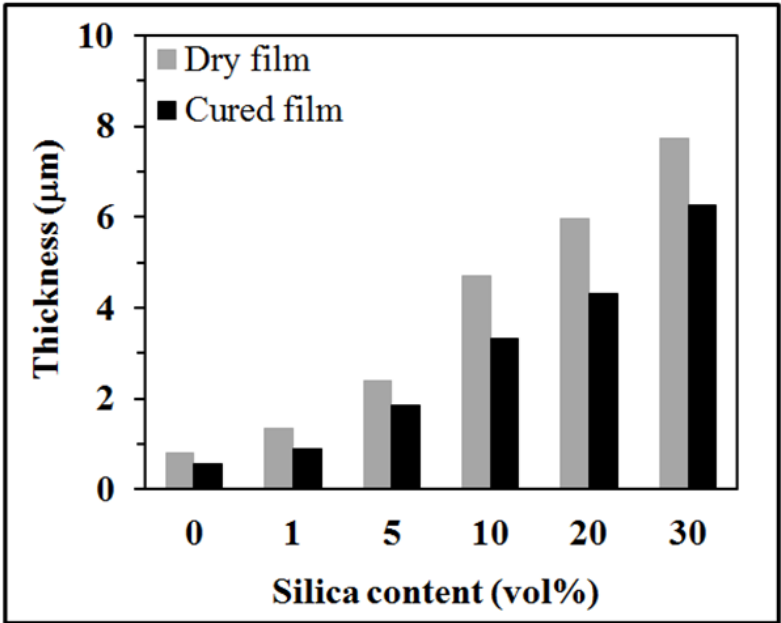

Fig. 2. Influence of silica content on the film thickness.

Fig. 2 shows the influence of the silica content on the film thickness. It can be seen that the average thickness of all the cured films is lower than that of all the dry films. Therefore, the time used for the UV-curing process is probably sufficient to transform the dry silica-acrylate films into the hardened UV-cured acrylate films. According its components, the UV-cured acrylate polymer is a high reactivity UV-curable resin, such as short reaction times on the order of seconds, offers a variety of monomers, and oligomers with functional end groups [15-16]. In addition, the UV intensity of the UV lamp used in this study was fixed at $110 \mathrm{~W}$. Consequently, no more shrinkage of the UV-cured acrylate film was detected though the UV-curing time was longer than $10 \mathrm{~min}$ [17-18]. Interestingly, the thickness of both the dry and cured films gradually increased when the silica content was increased. This is due to the increased amount of particles that produces more aggregates on the film surfaces [19]. This was confirmed by the results from the CLSM images at the magnification of 100x. The middle in position of the UV-cured acrylate films modified with the SSNPs was selected for observing the film surface. 

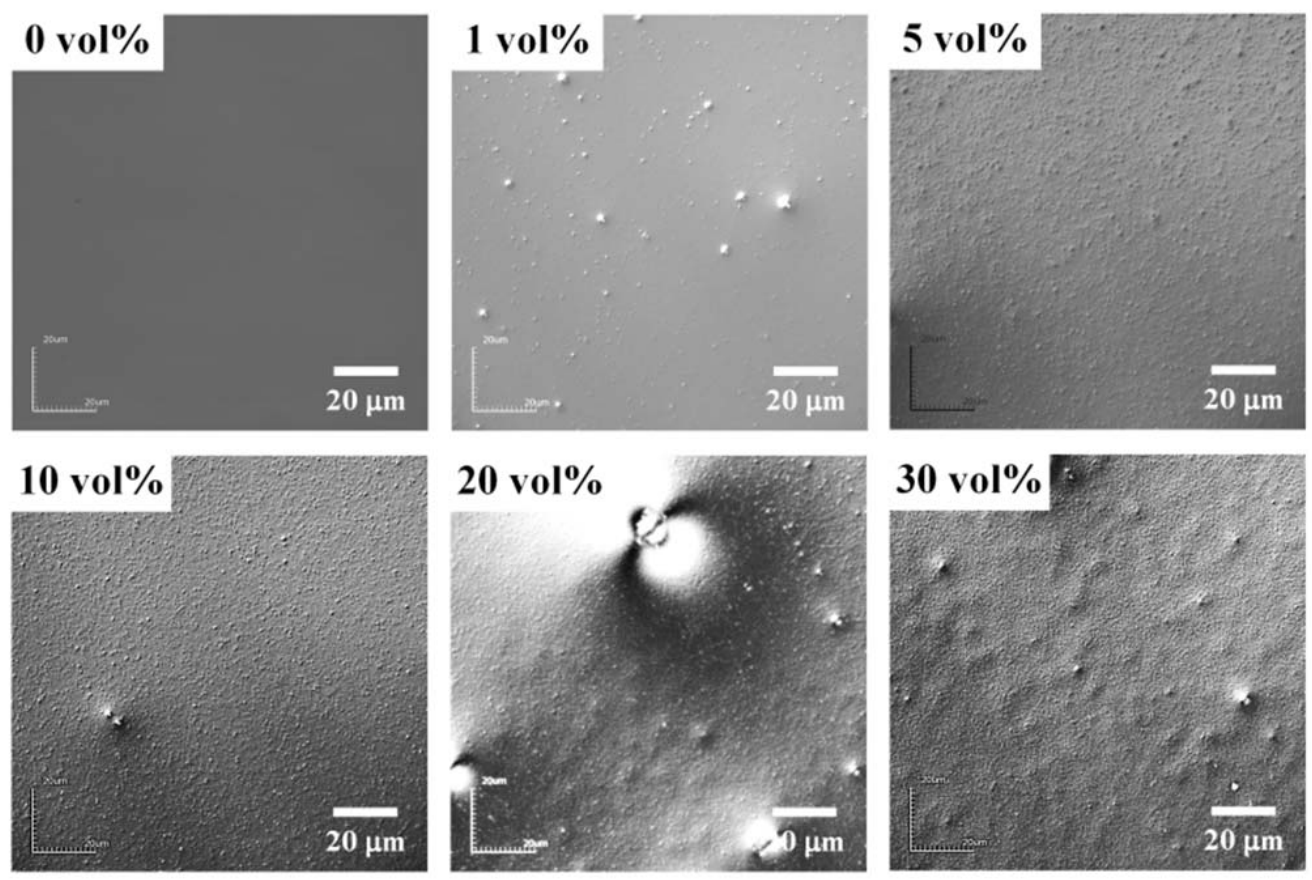

Fig. 3. CLSM images of the UV-cured acrylate films modified with the SSNPs.

Fig. 3 shows the CLSM images of the UV-cured acrylate films modified with the SSNPs. It was found that all the films coated on the glass substrates were uniform throughout the bar coating process. The unmodified film is smooth and no particles present. On the other hand, on the surface of all the modified films, it was observed that particles were clearly present. There is a low amount of particles on the film modified with 1 vol $\%$ SSNPs. The particles have a random dispersion. When the silica content was increased from 5 and 10 vol\%, the amount of the particles on the surface of the films moderately increased. Dispersibility of the particles on both films was quite good. The surface of these films seems to be rougher than the film modified with the 1 vol\% SSSNPs. Nonetheless, the surface roughness of the films was still less than that of the films modified with the 20 and $30 \mathrm{vol} \%$ SSNPs. The result of the thickness and roughness analyses of all the modified films are consistent with some previous studies, which reported that the increased silica content 
produces films with a high thickness and roughness [20-21]. The observed particles on the films modified with the 20 and 30 vol\% SSNPs have a larger size and more aggregates.

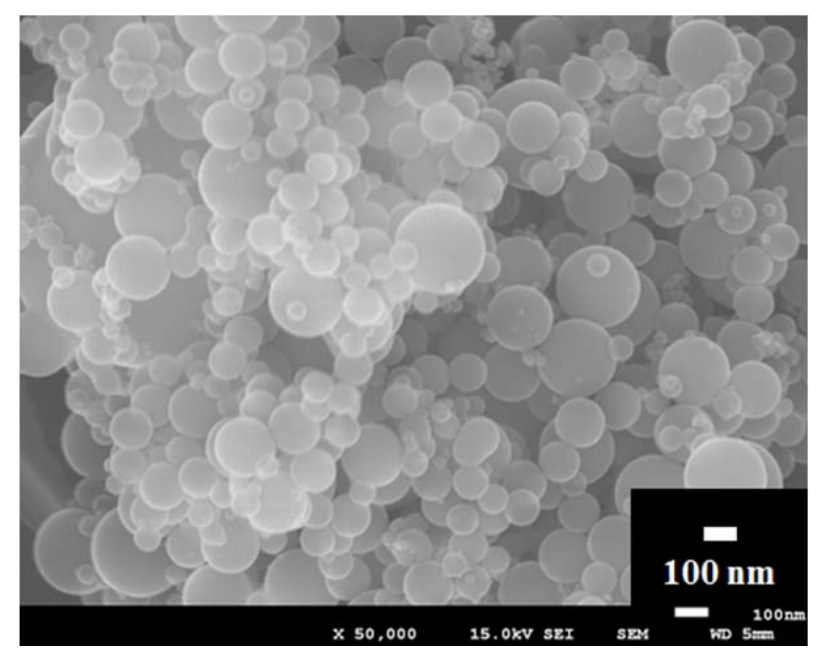

Fig. 4. FESEM image of the SSNPs as received.

However, visualization of the particles on all the modified films from the CLSM cannot accurately provide useful information about the size and form of the particles. It is due to some particles embedded in the films. Also, the magnification of the CLSM may be too low and insufficient to characterize when considering the original size of the SSNPs. The image reveals that all particles are totally spherical and the particle size is in the approximate range of $100-250 \mathrm{~nm}$.

An attempt to overcome these problems was made using the FESEM and the results are shown in Fig. 5. The SSNPs containing silicon (Si) atoms with a higher atomic number as compared to the carbon (C) atom in the polymer surface exhibit bright spot emissions. These are due to the COMPO mode that provides the image contrast as a function of the elemental composition [22]. The results clearly confirm the existence of the silica particles in the films. 

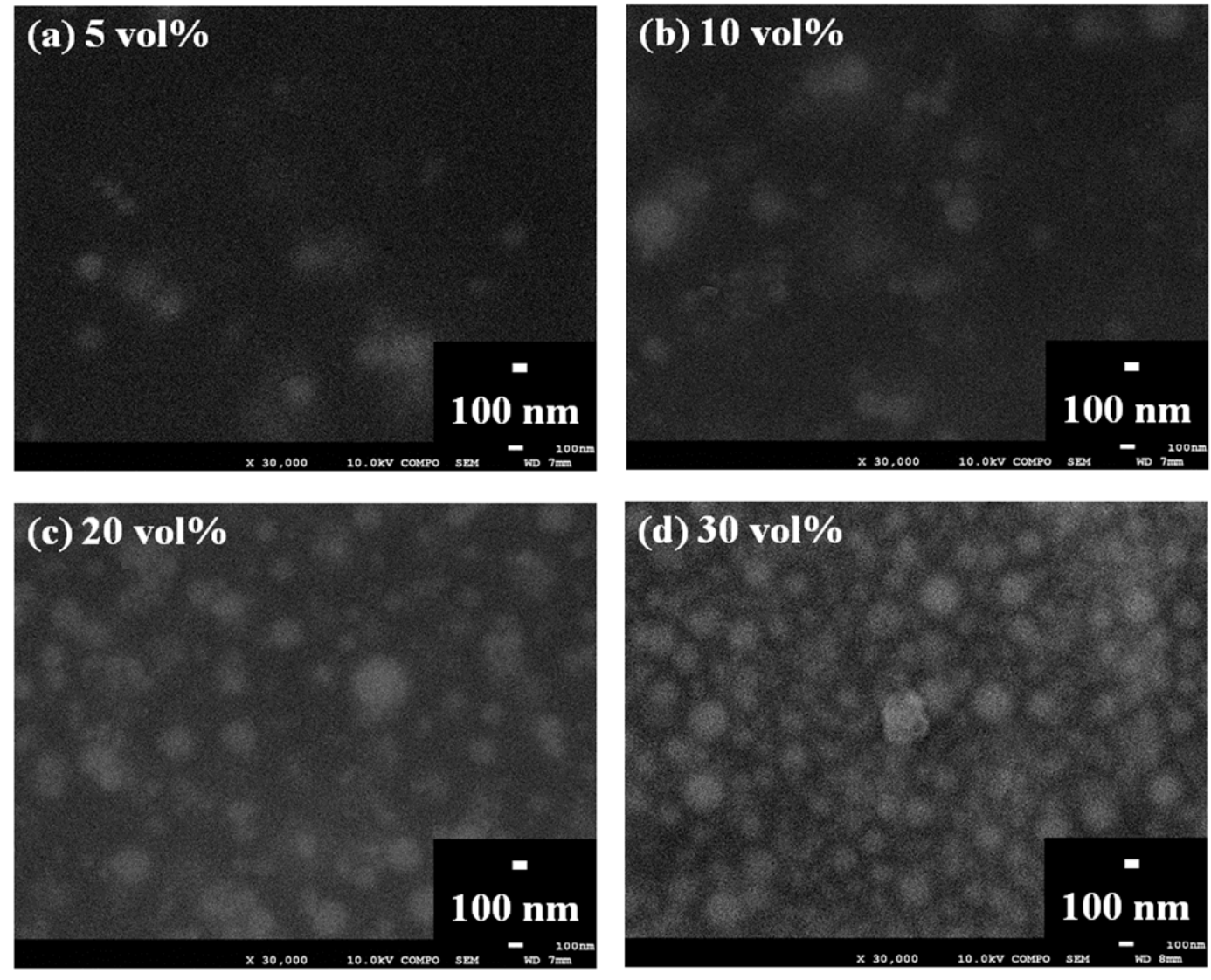

Fig. 5. FESEM images of the UV-cured acrylate films modified with various amounts of

$$
\text { SSNPs: (a) } 5 \text { vol } \% \text {, (b) } 10 \mathrm{vol} \% \text {, (c) } 20 \mathrm{vol} \% \text { and (d) } 30 \mathrm{vol} \% \text {. }
$$

Figs. 5 (a) and (b) illustrate the FESEM images of the film surfaces modified with 5 vol\% and 10 vol\% SSNPs; they show small amounts of the particles with random dispersions. The size of the observed particles is approximately $200 \mathrm{~nm}$ corresponding to the size of the received particles as shown in Fig. 4. In addition, some of the particles on these film surfaces are close together as aggregates. When the silica content was increased to 20 vol\%, the size of the aggregates is in the range of $300-500 \mathrm{~nm}$ as seen in Fig. 5 (c). This may be due to the fact that the SSNPs used here are hydrophilic and contains silanol groups on their surface leading to the formation of hydrogen bonds between them [23]. The distances 
between the aggregates decrease that is caused by the loss of the liquid carrier during the UVcuring process [19]. This behavior is also found in the FESEM image of the surface of the film modified with 30 vol\% SSNPs as illustrated in Fig. 5 (d). The image shows the smallest distances between the aggregates and the best dispersion of particles when compared to the other images of the film surfaces with the lower silica content. Moreover, it exhibits the highest amount of the particles and aggregates. These results indicate that the increased silica content possibly provides a high number of particles and a greater aggregation with a good dispersion leading to the increased thickness and roughness of the film. While, the increasing thickness and roughness of the films are responsible for the light diffusing ability of the films resulting in the enhanced haze value [24-25]. In addition, the presence of silica nanoparticles on the films can dramatically improve the optical properties of a polymer film because of their good light transmittance and diffusion capacity, which may be promising candidates for light diffusing films in the electronic display industry [12-13]. Thus, the optical properties of the UV-cured acrylate films modified with the SSNPs were studied and further discussed in the following section. 


\subsection{Optical properties of UV-cured acrylate films modified with spherical silica} nanoparticles

The transmittance of the UV-cured acrylate films modified with the SSNPs was measured by the UV-Vis spectrophotometer in the visible region and compared to the unmodified film.

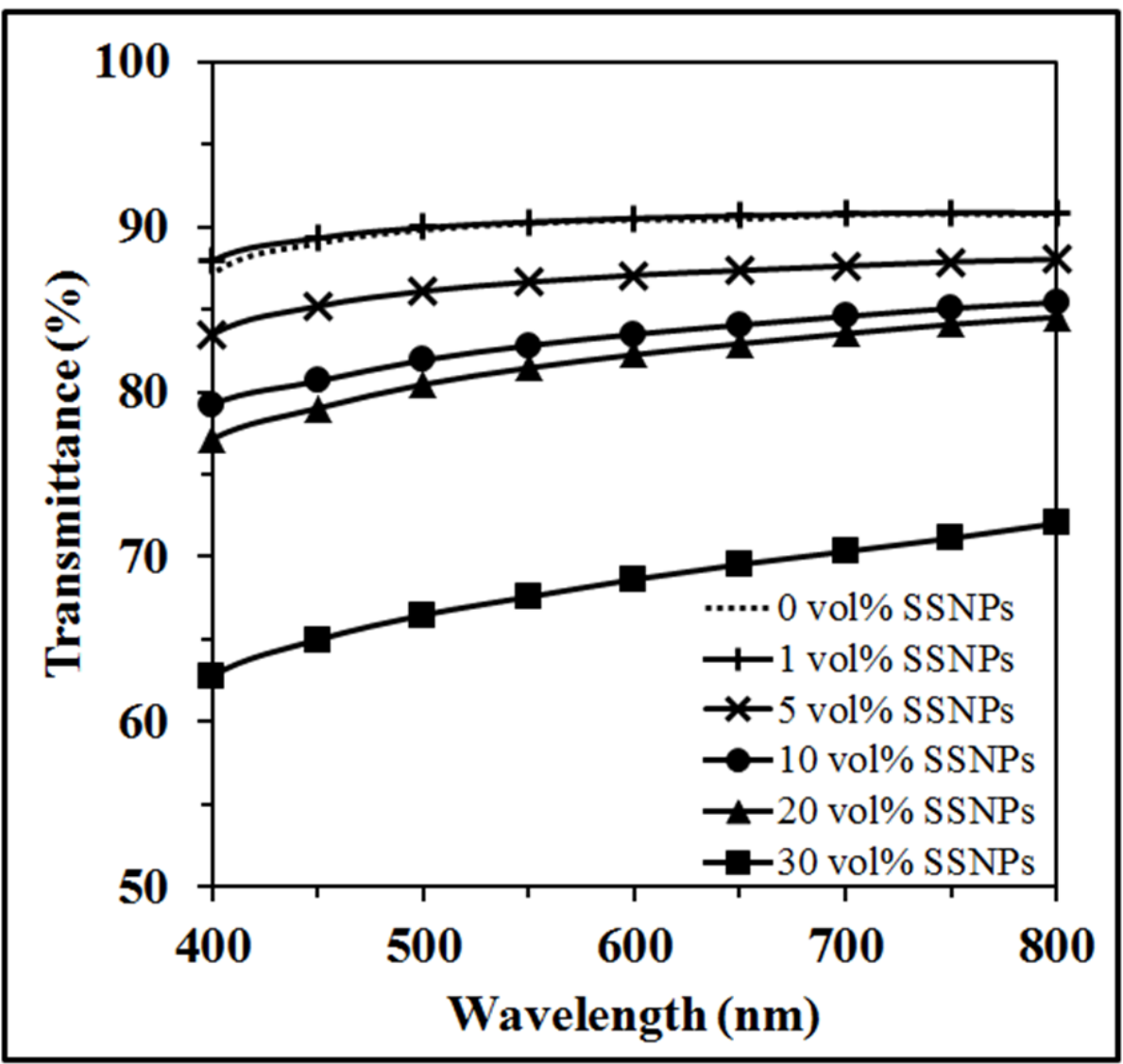

Fig. 6. Transmittance spectra of all films coated on glass substrates.

Fig. 6 illustrates the transmittance spectra of all films coated on the glass substrate as a function of the wavelength. The transmittance spectra of the UV-cured acrylate film modified with 1 vol\% overlap with the spectral characteristic of the unmodified film. Thus, the low silica content has no effect on the transmittance of the UV-cured acrylate film. In 
contrast, the transmittance of the modified films slightly decreases from 85 to $75 \%$ when the silica content was increased from 5 to $20 \mathrm{vol} \%$. The lowest transmittance obtained in the visible region was $60 \%$, which is observed from the film modified with the highest SSNPs (30 vol\%). These results suggest that the films become more opaque when the silica content was increased [26]. When light passes through heterogeneous films, the light scattering occurs due to the difference in the refractive indices between the heterogeneous phase (particles) and matrix [12]. Thus, the intensity of the light transmittance was attenuated. In principal, this type of light scattering is related to the size of the particles in the matrix [13]. If the size of the particles is much smaller than the wavelength of the incident light, the intensity of the light scatterting is inversely proportional to the fourth power of the wavelength. This light scattering type is called Rayleigh scattering. On the other hand, if the size of the particles is independent of the wavelength of the incident light, the light scattering is classified as Mie scattering. Based on the Mie scattering, the intensity of the light scattering is independent of the wavelength of the incident light. In addition, the direction of the light scattering goes toward that of the incident light. Interestingly, the transmittance of the films modified with the SSNPs slightly rises at a wavelength longer than $500 \mathrm{~nm}$, thus the intensity of the light scattering during the SSNPs is probably independent of the wavelength of the incident light. Therefore, the Mie scattering occurs in the films, and it can be inferred that the size of the particles or aggregated particles present inside the films is possibly larger than that of the wavelength of the incident light. Meanwhile, as received the particles are of various sizes with a high distribution (Fig. 4), and consequently, the various sizes of the observed particles on the film surfaces (Fig. 5) are almost smaller than $500 \mathrm{~nm}$ thus the Rayleigh scattering probably occurs on the film surfaces. These results are consistent with 
some previous studies [12]. It should be noted that the light scattering of the films corresponds to the Mie scattering interfering with the Rayleigh scattering.

The particle content in the films also plays a key role in their haze value, which is one of the main factors affecting the optical properties of the light diffusing films [12]. Therefore, the haze value of the UV-cured acrylate films modified with the SSNPs was measured by a UV-Vis spectrophotometer and then calculated in order to investigate the effect of the silica content on the haze value.

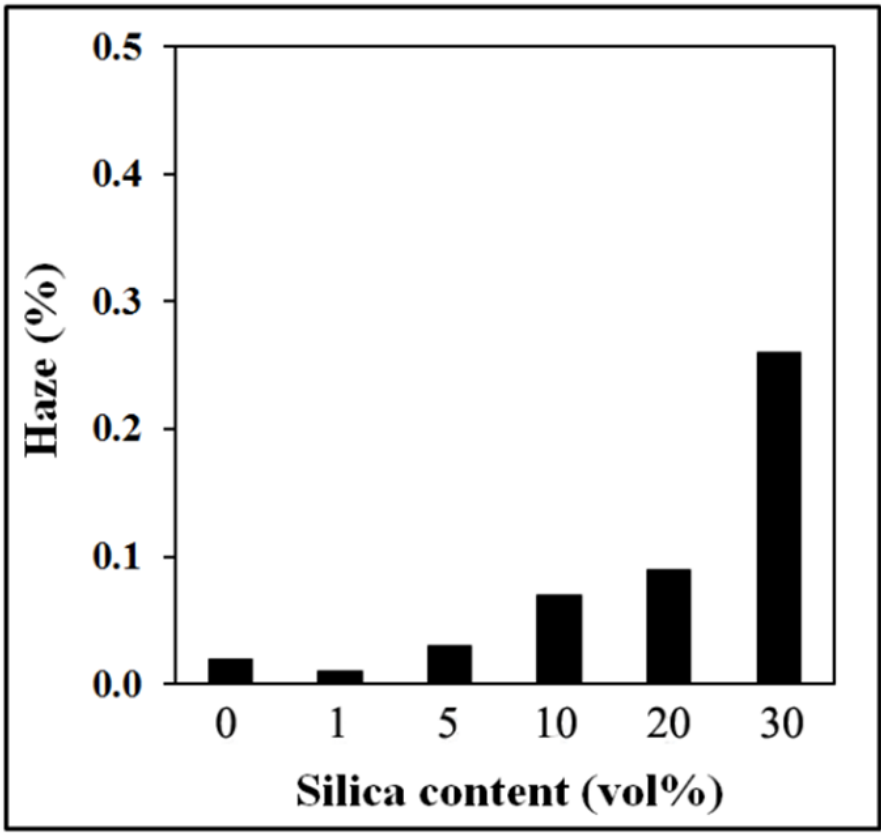

Fig. 7. Haze value of the UV-cured acrylate films modified with various silica contents.

Fig. 7 illustrates the haze value of the UV-cured acrylate films modified with various silica contents. The haze value slightly decreases as a result of the modification of the UVcured acrylate film with 1 vol\% SSNPs. On the other hand, the haze gradually increases when the silica content was greater than 5 vol\%. The film modified with 30 vol\% SSNPs has the highest haze value of about 0.26 although its transmittance is the lowest (as seen in Fig. 
6). This might be due to the haze value that is considered using the light diffusion that depends on the light scattering from the particles. When the light interacts with the high number of particles with a size comparable or larger than the wavelength of the light, it is more possible that the scattered light leads to the high light diffusing ability of the film [27].

The light diffusing ability of the UV-cured films modified with the SSNPs can be implied by their scattered light images. The scattered light images were visualized by the apparatus and subsequently taken by a digital camera.
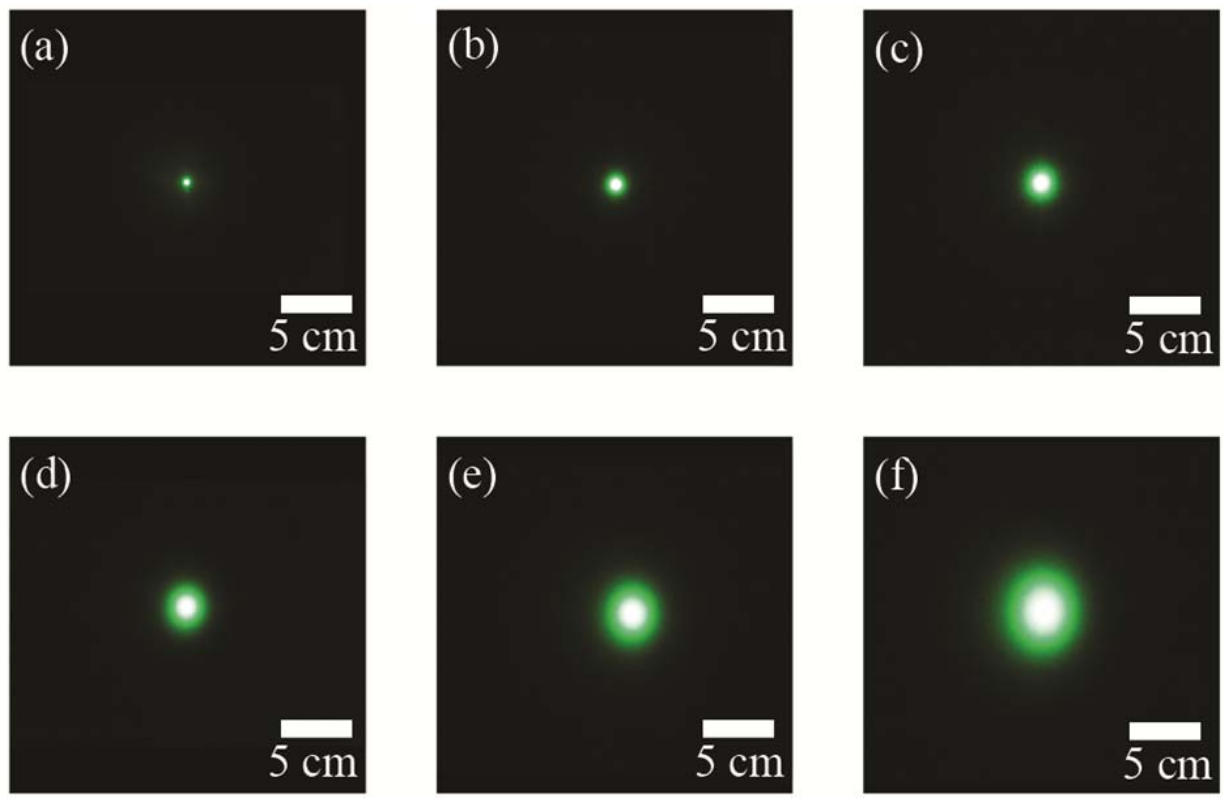

Fig. 8. The scattered light images of the UV-cured acrylate films containing various silica contents such as (a) 0 vol\% (UV-cured acrylate film), (b) 1 vol\%, (c) 5 vol\%, (d) 10 vol $\%$, (e) 20 vol $\%$ and (f) 30 vol $\%$.

The scattered light images of all the samples are displayed in Fig. 8. Fig. 8 (a) shows the scattered light image of the unmodified film, which seems to be slightly lower than that of the film modified with 1 vol\% SSNPs (Fig. 8 (b)). The range of the scattered light by the particles in the film modified with 1 vol\% SSNPs is somewhat smaller by about five times 
than that of the films modified with silica of about 10 and 20 vol\% as seen in Figs. 8 (c) and (d). The largest range of the scattered light by the particles is clearly observed in Fig. 8 (f), which shows the scattered light image of the film modified with the highest silica content (30 vol\%). Based on the above mentioned results, it can be inferred that the light diffusing ability of the UV-cured acrylate film increased when the silica content increased. This is due to the large number of silica nanoparticles that probably produce a high light scattering resulting in the enhancement of their light diffusing ability that corresponds to the haze value [12]. When the polymer matrix is assumed to be tranparent and homogeneous, the particles are only assumed to produce the light scattering [27]. Moreover, the observed particles on the film surface as evidenced from the FE-SEM images (Fig. 5) seem to be close to each other. Thus, it can be implied that each particle is more illuminated by both the incident light and the secondary scattering than from other particles when the light interacts with the large amount of particles of a size comparable or larger than the wavelength of light. This behavior is called multiple scattering based on the Mie theory. The multiple scattering usually occurs when an aggregate is obtained in the film. Moreover, the aggregate does not have enough separation thus the scattered light of each particle or cluster is assumed to be dependent on each other. In addition, the scattered light continuously occurs in a film with a homogeneous dispersion of particles corresponding to the morphology of the UV-cured film with the high silica loading of $30 \mathrm{vol} \%$ presented in Figure 5 (d). It is note worthy that the image of the unmodified film (UV-cured acrylate film) in Fig. 8 (a) showed no light scattering effect. In addition, a more uniform distribution of scattered light was obtained with the UV-cured polaycrylic films modified with the SSNPs. These results further prove the advantage of the SSNPs for improving the light diffusing property of the UV-cured acrylate films. Thus, the films prepared in this study are promising candidates for light diffusing films. 


\section{Conclusion}

The UV-cured acrylate films modified with the SSNPs was successfully fabricated by coating suspension of silica-acrylate on cleaned substrates. The increasing silica content in the films reduces the transmittance of the films. The increasing silica content also provides a good compatibility and good dispersion of the particles which are some of the main ways to improve the optical properties of the films. The modified films have both a high haze and large range of scattered light. The largest range of the scattered light (transmittance $60 \%$ and haze 0.26 ) was obtained from the film modified with 30 vol\% SSNPs. The spherical silica nanoparticles may be utilized in the polymer films to obtain a high diffusing ability that is useful in the field of electronic display technologies.

\section{Acknowledgements}

This study was supported by the Advanced Low Carbon Technology Research and Development Program (ALCA) of the Japan Science and Technology Agency (JST).

\section{References}

[1] Nanofabrication: Principle, Capabilities and Limits, Springer Technology \& Engineering, New York, 2009, pp. 182.

[2] J.F. Rabek, Radiation Curing in Polymer Science and Technology, Elsevier Applied Science, London, 1993. 
[3] L. Keller, C. Decker, K. Zahouily, S. Benfarhi, J.M. Le Meins, J. Miehe-Brendle, Synthesis of polymer nanocomposites by UV-curing of organoclay-acrylic resins, Polymer 45 (2004) $7437-7447$.

[4] R. Peters, V.M. Litvinov, P. Steeman, A.A. Dias, Y. Mengerink, R. van Benthem, C.G. de Koster, Sj. van der Wal, P. Schoenmakers, Characterisation of UV-cured acrylate networks by means of hydrolysis followed by aqueous size-exclusion combined with reversed-phase chromatography, J. Chromatogr. A 1156 (2007) 111 - 123.

[5] A. Milinavičiūtè, V. Jankauskaitè, P. Narmontas, Mater. Sci. (Medžiagotyra), Properties of UV-curable hyperbranched urethane-acrylate modified acrylic monomer coatings, Mater. Sci. (Medžiagotyra) 7 (2011) 378 - 383.

[6] C.J. Chang, M.H. Tsai, P.C. Kao, H.Y. Tzeng, Optical and mechanical properties of jet printed and UV cured blue pixels with phosphated epoxy acrylate as the curing agent, Thin Solid Films 516 (2008) 5503 - 5507.

[7] K. Ohashi, K. Nakai, S. Kitayama, T. Kawaguchi, Mechanisms of hydrogen evolution and stabilization of UV-cured urethane acrylate resin for coating of optical fiber, Polym. Degrad. Stab. 22 (1988) 223 - 232.

[8] M. Giamberini, G. Malucelli, Hybrid organic-inorganic UV-cured films containing liquid-crystalline units, Thin Solid Films 548 (2013) 150 - 156.

[9] S. Song, Y. Sun, Y. Lin, B. You, A facile fabrication of light diffusing film with LDP/polyacrylates composites coating for anti-glare LED application, Appl. Surf. Sci. 273 (2013) $652-660$. 
[10] X.Y. Shang, Z.K. Zhu, J. Yin, and X.D. Ma, Compatibility of soluble polyimide/silica hybrids induced by a coupling agent, Chem. Mater. 14 (2002) $71-77$.

[11] Y. Wang, H. Wang, X. Meng, R. Chen, Antireflective films with Si-O-P linkages from aqueous colloidal silica: Preparation, formation mechanism and property, Sol. Energy Mater. Sol. Cells 130 (2014) 71 - 82.

[12] S. Guo, S. Zhou, H. Li, B. You, Light diffusing films fabricated by strawberry-like PMMA/SiO 2 composite microspheres for LED application, J. Colloid Interface Sci 448 (2015) $123-129$.

[13] J. Hu, Y. Zhou, M. He, X. Yang, Novel multifunctional microspheres of polysiloxane@ $\mathrm{CeO}_{2}$-PMMA: Optical properties and their application in optical diffusers, Opt. Mater. 36 (2013) 271 - 277.

[14] Q.J. Jiang, J.G. Lu, J. Zhang, Y.L. Yuan, H. Cai, L. Hu, L.S. Feng, B. Lu, X.H. Pan, Z.Z. Ye, Texture-etched broad surface features of double-layered $\mathrm{ZnO}: \mathrm{Al}$ transparent conductive films for high haze values, J. Alloys Compd. 596 (2014) 107 - 112.

[15] C. Decker, UV - radiation curing chemistry, Pigment Resin Technol., 30 (2001) 278.

[16] L. E. Schmidt, Y. Leterrier, J.-M. Vesin, M. Wilhelm, J.-A. E. Månson, Photorheology of fast UV-curing multifunctional acrylates, Macromol. Mater. Eng. 290 (2005) 1115.

[17] S. S. Lee, A. Luciani, J.-A. E. Månson, A rheological characterisation technique for fast UV-curable systems, Prog. Org. Coat. 38 (2000) 193. 
[18] P. A. M. Steeman, A. A. Dias, D. Wienke, T. Zwartkruis, Polymerization and network formation of UV-curable systems monitored by hyphenated real-time dynamic mechanical analysis and near-infrared spectroscopy, Macromolecules 37 (2004) 7001.

[19] M. Sumiya, K. Yamada, Development of filterability test method for gel retention performance for UV curable ink jet inks, in: Proc. NIP30: The 30th International Conference on Digital Printing Technologies and Digital Fabrication, PA, United States, 2014.

[20] W. Ming, D. Wu, R.V., Benthem, Superhydrophobic films from raspberry-like particles, Nano Lett. 5 (2005) 2298 - 2301.

[21] K. Senoo, Structure analysis of nanoparticle dispersed in transparent resin using synchrotron X-ray scattering, Spring 8 Research Frontiers 2010 in Industrial Applications (2010) $136-137$.

[22] Y.J. Shin, S.H. Kim, D.H. Yang, H. Kwon, J.S. Shin, Amperometric glucose biosensor by means of electrostatic layer-by-layer adsorption onto polyaniline-coated polyester films, J. Ind. Eng. Chem., 16 (2010) 380 - 384.

[23] T. Nazir, A. Afzal, H.M. Siddiqi, Z. Ahmad, M. Dumon, Thermally and mechanically superior hybrid epoxy-silica polymer films via sol-gel method, Prog. Org. Coat. 69 (2010) $100-106$.

[24] Q. Gao, H. Jiang, C. Li, Y. Ma, X. Li, Z. Ren, Y. Liu, C. Song, G. Han, Tailoring of textured transparent conductive $\mathrm{SnO}_{2}: \mathrm{F}$ thin films, J. Alloys Compd. 574 (2013) 427 - 431.

[25] X. Yan, S. Venkataraj, A.G. Aberle, Wet-chemical surface texturing of sputterdeposited $\mathrm{ZnO}$ :Al films as front electrode for thin-film silicon solar cells, Int. J. Photoenergy (2015). 
[26] B.Ö. Uysal, F.Z. Tepehan, Controlling the growth of particle size and size distribution of silica nanoparticles by the thin film structure, J Sol-Gel Sci Technol 63 (2012) 177 - 186.

[27] A. Rastar, M.E. Yazdanshenas, A. Rashidi, S.M. Bidoki, Theoretical review of optical properties of nanoparticles, J. Eng. Fiber Fabr. 8 (2013) 85 - 96. 